\title{
Trends in inflammatory bowel disease therapy
}

F ALK SYMPOSIUM NO. 56, 'TRENDS IN INFLAMMATORY bowel disease therapy', comprised eight sessions over three days and attracted more than 400 participants.

The first session, 'Pathogenesis: Clues for future therapy', involved seven speakers as well as an overview summary. Considerable new information was presented in a very lucid and well organized manner; in particular, each speaker referred to a prior or forthcoming presentation, so that the whole panel left an impression of cohesion. The pathogenesis session included aspects of immunogenetics, antigen presentation, gut microflora, immune-mediated cytotoxicity, the role of mast cells, inflammatory mediators, and intestinal oxidative damage.

The second session concerned the diagnosis of inflammatory bowel disease and involved six speakers who covered diagnostic imaging, endoscopic and pathological diagnosis, nuclear medicine scans, differential diagnosis with audience participation, and an assessment of new technology for assessing the outcome of severe ulcerative colitis.

The third session addressed 'Lifestyle issues: What do I tell my patients?' and involved presentations on genetic counselling, smoking, diet, oral contraceptives, sex, pregnancy, breast feeding, counselling for the pediatric patient and a planned long term approach to patient management.

The fourth session, on carcinogenesis, included contributions from six speakers and the usual overview. Topics included experimental animal studies, dysplasia, the role of oncogenes and the use of flow cytometry. There was a special presentation on surveillance programs in ulcerative colitis with a proponent of this and a counter presentation, "Is surveillance worthwhile?" which provoked vigorous, healthy discussion.

A short session on nutritional and pediatric therapy comprised two speakers, with a talk on the state-of-the-art of nutritional therapy in Crohn's disease, and pediatric therapy of inflammatory bowel disease.

The sixth session, 'Trends in medical therapy', was well covered by six speakers. Topics included the newer oral preparations, budesonide and fluticasone, in ulcerative colitis, followed by a discussion of coated omega- 3 fatty acids as adjunctive therapy in ulcerative colitis. The role of cyclosporine in steroid-resistant Crohn's disease and the use of colonoscopy to optimize steroid treatment dosing and duration in Crohn's disease was also discussed.

The seventh session concerned surgical therapy, with four presentations covering pouch surgery and the importance of the transitional zone, motility and compliance, pouchitis and the return of ileorectal anastomosis.

The final session was devoted to the emerging role of new salicylates in inflammatory bowel disease therapy. There were presentations by 13 speakers, mostly of original work.

Forty-five manuscripts were received and accepted for this issue, including two from the poster session.

L Sutherland, F Martin, R Farmer, G Hellers, CN Williams Scientific Organizing Committee 


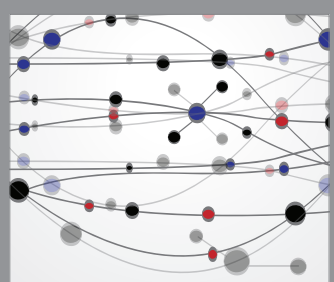

The Scientific World Journal
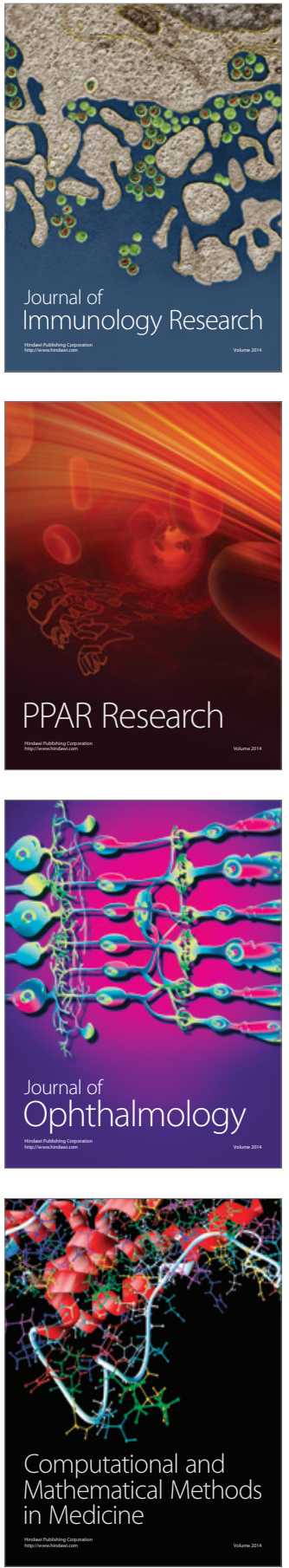

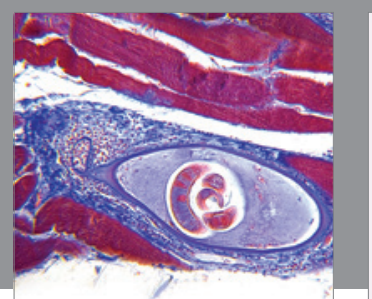

Gastroenterology Research and Practice

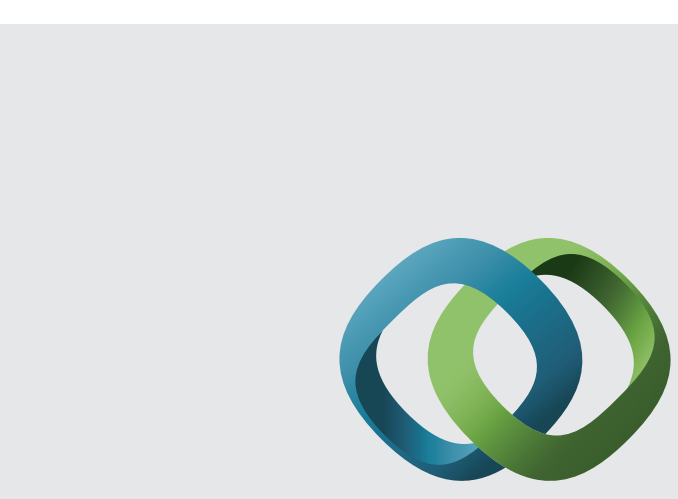

\section{Hindawi}

Submit your manuscripts at

http://www.hindawi.com
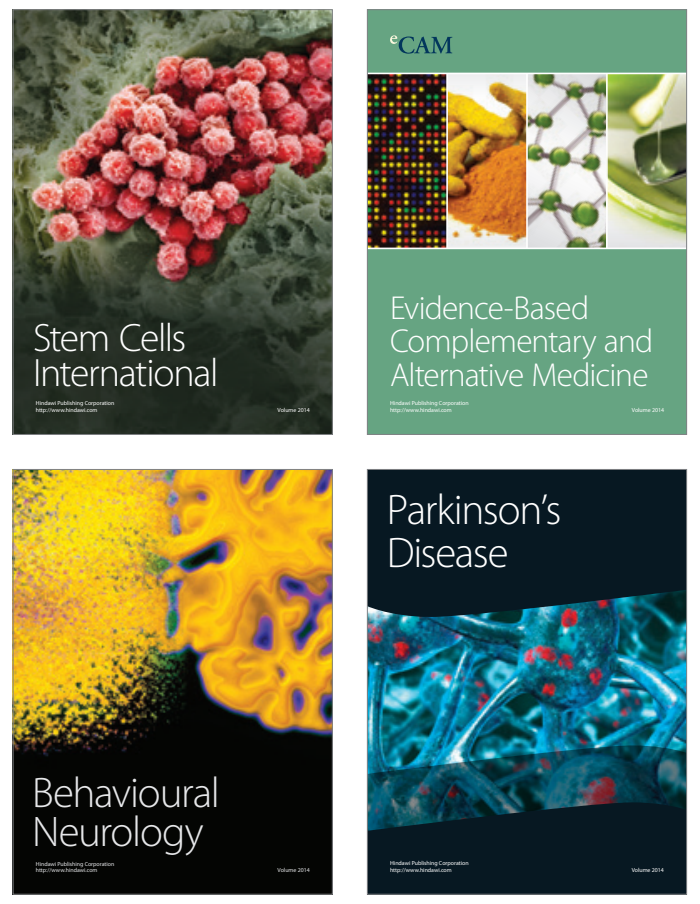
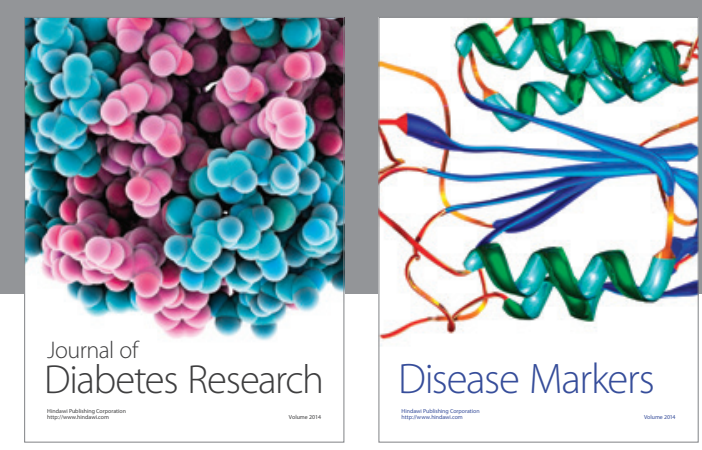

Disease Markers
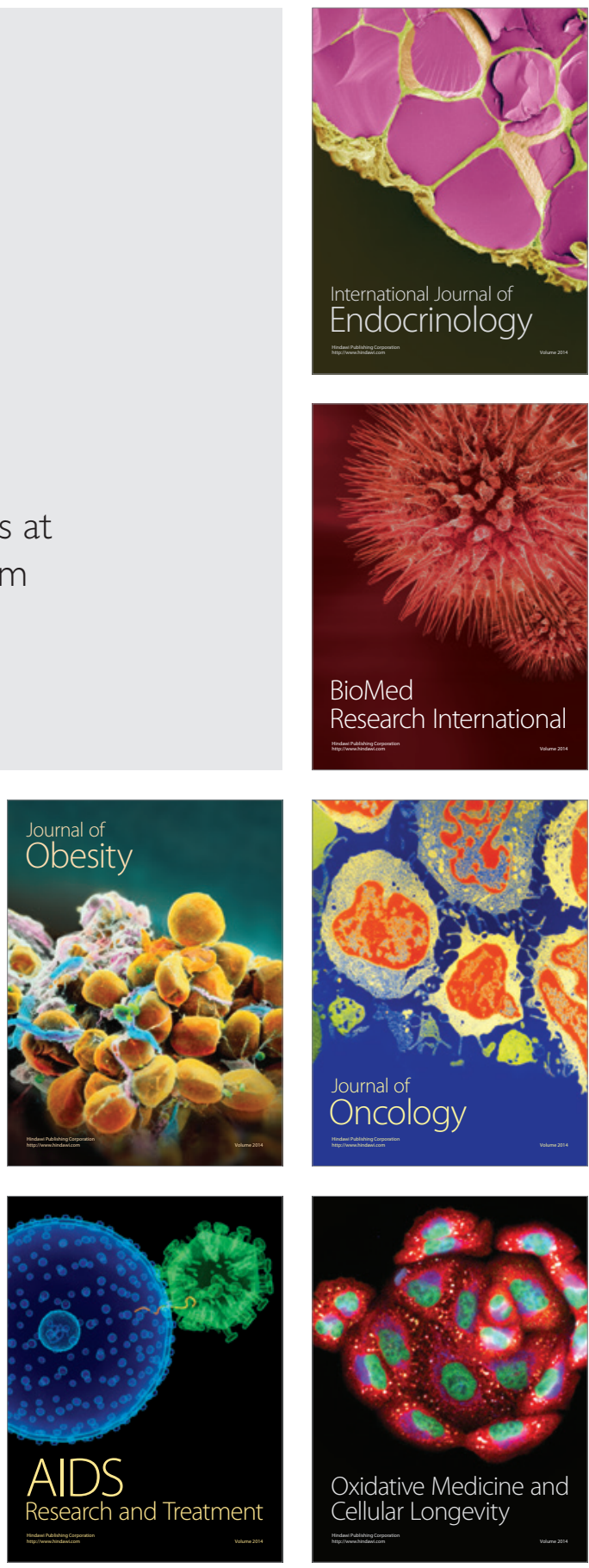